\title{
65.
}

\section{NOUVELLES RECHERCHES SUR LES FONCTIONS DE M. STURM.}

[From the Journal de Mathématiques Pures et Appliquées (Liouville), tom. XIII. (1848), pp. 269-274.]

EN développant une remarque faite par M. Sylvester dans un Mémoire publié il y a huit ou neuf ans dans le Philosophical Magazine, j'ai trouvé des expressions assez simples des fonctions de M. Sturm, composées au moyen des coefficients mêmes; ce qui convient mieux que d'exprimer ces fonctions, comme je l'ai déjà fait dans le t. xI. de ce Journal, p. 297, [48], par les sommes des puissances. D'ailleurs il ne m'est plus nécessaire de parler des expressions de M. Sylvester, ou même des divisions successives de M. Sturm; mais ma méthode fait voir directement que les fonctions que je vais définir sont douées de la propriété fondamentale sur laquelle se repose la théorie de M. Sturm, à savoir que, en considérant trois fonctions successives, la première et la dernière fonction sont de signe contraire pour toute valeur de la variable qui fait évanouir la fonction intermédiaire; cependant je n'ai pas encore réussi à démontrer dans toute sa généralité l'équation identique d’où dépend cette propriété.

Soient d'abord $V, V^{\prime}$ des fonctions du même degré $n$,

$$
\begin{aligned}
& V=a x^{n}+b x^{n-1}+\ldots, \\
& V^{\prime}=a^{\prime} x^{n}+b^{\prime} x^{n-1}+\ldots,
\end{aligned}
$$

et écrivons

$$
\begin{aligned}
-F_{1} & =\left|\begin{array}{cc}
V, & V^{\prime} \\
a & a^{\prime}
\end{array}\right|, \\
F_{2} & =\left|\begin{array}{cccc}
x V, & V, & x V^{\prime}, & V^{\prime} \\
a & \cdot & a^{\prime} & \cdot \\
b & a & b^{\prime} & a^{\prime} \\
c & b & c^{\prime} & b^{\prime}
\end{array}\right|,
\end{aligned}
$$




$$
-F_{3}=\left|\begin{array}{cccccc}
x^{2} V, & x V, & V, & x^{2} V^{\prime}, & x V^{\prime}, & V^{\prime} \\
a & \cdot & . & a^{\prime} & \cdot & . \\
b & a & . & b^{\prime} & a^{\prime} & . \\
c & b & a & c^{\prime} & b^{\prime} & a^{\prime} \\
d & c & b & d^{\prime} & c^{\prime} & b^{\prime} \\
e & d & c & e^{\prime} & d^{\prime} & c^{\prime}
\end{array}\right| \text { c. }
$$

(ce qui suffit pour faire voir la loi de ces fonctions successives $F_{1}, F_{2}, \ldots$ ). Il résulte des propriétés élémentaires des déterminants que ces fonctions sont des ordres $\overline{n-1}$, $\overline{n-2}, \overline{n-3}$, \&c., respectivement, par rapport à la variable $x$. En effet, dans $F_{1}$ le coefficient de $x^{n}$ se réduit à $\left|\begin{array}{ll}a, & a^{\prime} \\ a & a^{\prime}\end{array}\right|$, savoir, à zéro; de même, dans $F_{2}$, les coefficients de $x^{n}$ et $x^{n-1}$ se réduisent chacun à zéro; et ainsi de suite.

Soient encore

$$
\begin{aligned}
& P_{1}=\left|\begin{array}{ll}
a, & a^{\prime} \\
b & b^{\prime}
\end{array}\right|, \\
& P_{1}^{\prime}=\left|\begin{array}{ll}
a, & a^{\prime} \\
c & c^{\prime}
\end{array}\right|, \\
& P_{2}=\left|\begin{array}{llll}
a, & . & a^{\prime}, & \cdot \\
b & a & b^{\prime} & a^{\prime} \\
c & b & c^{\prime} & b^{\prime} \\
d & c & d^{\prime} & c^{\prime}
\end{array}\right| \\
& P_{2}^{\prime}=\left|\begin{array}{llll}
a & . & a^{\prime}, & . \\
b & a & b^{\prime} & a^{\prime} \\
c & b & c^{\prime} & b^{\prime} \\
e & d & e^{\prime} & d^{\prime}
\end{array}\right| \\
& P_{3}=\quad a, \quad . \quad a^{\prime}, \quad \cdot \quad, \quad \& c . \\
& P_{3}^{\prime}=a, \quad . \quad a^{\prime}, \quad . \quad \text {, \&c. } \\
& \begin{array}{llllll}
b & a
\end{array} b^{\prime} \quad a^{\prime} . \\
& \begin{array}{llllll}
c & b & a & c^{\prime} & b^{\prime} & a^{\prime}
\end{array} \\
& \begin{array}{llllll}
d & c & b & d^{\prime} & c^{\prime} & b^{\prime}
\end{array} \\
& \begin{array}{llllll}
e & d & c & e^{\prime} & d^{\prime} & c^{\prime}
\end{array} \\
& \begin{array}{llllll}
f & e & d & f^{\prime} & e^{\prime} & d^{\prime}
\end{array} \\
& \begin{array}{lllllll}
b & a & b^{\prime} & a^{\prime}
\end{array} \text {. } \\
& \begin{array}{llllll}
c & b & a & c^{\prime} & b^{\prime} & a^{\prime}
\end{array} \\
& \begin{array}{llllll}
d & c & b & d^{\prime} & c^{\prime} & b^{\prime}
\end{array} \\
& \begin{array}{llllll}
e & d & c & e^{\prime} & d^{\prime} & c^{\prime}
\end{array} \\
& \begin{array}{llllll}
g & f & e & g^{\prime} & f^{\prime} & e^{\prime}
\end{array}
\end{aligned}
$$

(ce qui suffit pour indiquer la loi). On aura entre ces différentes fonctions $F, P, P^{\prime}$ cette suite remarquable d'équations identiques,

$$
\begin{aligned}
& P_{1}^{2} F_{3}+\left(x P_{1} P_{2}+P_{1} P_{2}^{\prime}+P_{1}^{\prime} P_{2}\right) F_{2}+P_{2}^{2} F_{1}=0, \\
& P_{2}^{2} F_{4}+\left(x P_{2} P_{3}+P_{2} P_{3}^{\prime}+P_{2}^{\prime} P_{3}\right) F_{3}+P_{3}^{2} F_{2}=0, \\
& \quad \text { \&c. }
\end{aligned}
$$

lesquelles équations, dans ce Mémoire, seront prises pour vraies. Cela étant, il est évident que $F_{1}$ et $F_{3}$ seront de signe contraire pour tonte valeur de $x$ qui fait évanouir $F_{2} ; F_{2}$ et $F_{4}$ seront de signe contraire pour toute valeur de $x$ qui fait évanouir $F_{3}$; et ainsi de suite.

c. 
Ces formules renferment le cas où les deux fonctions $V, V^{\prime}$ ne sont pas du même degré (en effet, pour les y adapter, on n'a besoin que de faire évanouir quelques-uns des premiers coefficients de $V$ ou de $V^{\prime}$ ). Il est donc permis de supposer que $V^{\prime}$ soit la dérivée de $V$. Dans ce cas, $F_{1}=a V^{\prime}$, et on verra dans un moment que les fonctions $F_{2}, F_{3}, \ldots$ contiennent chacune le facteur $a^{2}$, de manière qu'il convient d'écrire $F_{2}=a^{2} V_{2}$, $F_{3}=a^{2} V_{3}, \ldots$. Ce facteur $a^{2}$ peut être évidemment écarté, et ce sera de même avec le facteur $a$ de $F$, pourvu, ce que je supposerai dans la suite, que $a$ soit positif. On aura de cette manière les fonctions $V, V^{\prime}, V_{2}, V_{3}, \ldots$ douées des propriétés des fonctions de M. Sturm. En effet, elles seront précisément les fonctions $f x, f_{1} x, f_{2} x, \ldots$ du Mémoire déjà cité, ce qui cependant pourrait être difficile à démontrer à priori.

On déduit tout de suite des expressions de $F_{2}, F_{3}, \ldots$,

$$
a V_{2}=-\left|\begin{array}{ccc}
V, & x V^{\prime}, & V^{\prime} \\
a & n a & . \\
b & \overline{n-1} b & n a
\end{array}\right|, \quad a V_{3}=\left|\begin{array}{ccccc}
x V, & V, & x^{2} V^{\prime}, & x V^{\prime}, & V^{\prime} \\
a & . & n a & . \\
b & a & \overline{n-1} b & n a & . \\
c & b & \overline{n-2} c & \overline{n-1} b & n a \\
d & c & \overline{n-3} d & \overline{n-2} c & \overline{n-1} b
\end{array}\right| \text {, }
$$

Ces formules se simplifient au moyen des propriétés connues des déterminants, et en écrivant

$$
x V^{\prime}-n V=-U
$$

cela donne

$$
a V_{2}=\left|\begin{array}{ccc}
V, & U, & V^{\prime} \\
a & \cdot & \cdot \\
b & b & n a
\end{array}\right|, \quad a V_{3}=\left|\begin{array}{ccccc}
x V, & V, & x U, & U, & V^{\prime} \\
a & \cdot & \cdot & \cdot & \cdot \\
b & a & b & . & . \\
c & b & 2 c & b & n a \\
d & c & 3 d & 3 c & \overline{n-1} b
\end{array}\right|, \text { \&c. }
$$

ou enfin, et en écrivant un autre terme de la suite, afin de mieux faire voir la loi,

$$
\begin{aligned}
& V_{2}=-\left|\begin{array}{cc}
U, & V^{\prime} \\
b & n a
\end{array}\right|, \quad V_{3}=-\left|\begin{array}{cccc}
V, & x U, & U, & V^{\prime} \\
a & b & . & . \\
b & 2 c & b & n a \\
c & 3 d & 2 c & \overline{n-1} b
\end{array}\right| \\
& V_{4}=-\left|\begin{array}{ccccc}
x V, & V, & x^{2} U, & x U, & V^{\prime} \\
a & . & b & . & .
\end{array}\right|, \& c . \\
& \begin{array}{ccccc}
b & a & 2 c & b & . \\
c & b & 3 d & 2 c & n a
\end{array} \\
& \begin{array}{ccccc}
c & b & 3 d & 2 c & n a \\
d & c & 4 e & 3 d & \overline{n-1} b \\
e & d & 5 f & 4 e & \overline{n-2} c
\end{array} \mid
\end{aligned}
$$


formules dans lesquelles

$$
\left\{\begin{array}{l}
V=a x^{n}+b x^{n-1}+c x^{n-2}+\ldots \\
V^{\prime}=\quad n a x^{n-1}+n-1 b x^{n-2}+\ldots \\
U=\quad b x^{n-1}+2 c x^{n-2}+\ldots
\end{array}\right.
$$

et où, en substituant ces valeurs, on peut commencer pour $V_{2}$ avec le terme qui contient $x^{n-2}$, pour $V_{3}$ avec le terme qui contient $x^{n-3}$, et ainsi de suite, puisque les termes des ordres plus hauts s'évanouissent identiquement. Voilà, je crois, les expressions les plus simples des fonctions de M. Sturm.

Je donnerai en conclusion ces formes développées des fonctions jusqu'à $V_{4}$.

$$
\begin{aligned}
& V=a x^{n}+b x^{n-1}+c x^{n-2}+\ldots ; \\
& V^{\prime}=n a x^{n-1}+\overline{n-1} b x^{n-2}+\overline{n-2} c x^{n-3}+\ldots ; \\
& V_{2}=-n a\left\{2 c x^{n-2}+3 d x^{n-3}+\ldots\right. \\
& +b\left\{\overline{n-1} b x^{n-2}+\overline{n-2} c x^{n-3}+\ldots\right. \text {; } \\
& V_{3}=\left[2 n a b c-\overline{n-1} b^{3}\right. \\
& +\left[-2 n^{2} a c+\overline{n-1} a b^{2} \quad\right]\left\{4 e x^{n-3}+5 f x^{n-4}+\ldots\right. \\
& +\left[3 n a^{2} d-(3 n-2) a b c+(n-1) b^{3}\right]\left\{3 d x^{n-3}+4 e x^{n-4}+\ldots\right. \\
& +\left[-3 a b d+4 a c^{2}-b^{2} c \quad\right]\left\{(n-2) c x^{n-3}+(n-3) d x^{n-4}+\ldots\right. \text {; } \\
& V_{4}=A\left\{f x^{n-4}+g x^{n-5}+\ldots\right. \\
& +B\left\{e x^{n-4}+f x^{n-5}+\ldots\right. \\
& +C\left\{6 g x^{n-4}+7 h x^{n-5}+\ldots\right. \\
& +D\left\{5 f x^{n-4}+6 g x^{n-5}+\ldots\right. \\
& +E\left\{4 e x^{n-4}+5 f x^{n-5}+\ldots\right. \\
& +F\left\{\overline{n-3} d x^{n-4}+\overline{n-4} e x^{n-5}+\ldots ;\right.
\end{aligned}
$$

dans cette dernière expression j'ai mis, pour abréger,

$$
\begin{aligned}
A= & 9 n a^{2} b d^{2}-8 n a^{2} b c e+(4 n-4) a b^{3} e-(10 n-12) a b^{2} c d \\
& +(4 n-8) a b c^{3}-(n-2) b^{3} c^{2}+(2 n-2) b^{4} d, \\
B= & 10 n a^{2} b c f-12 n a^{2} b d e-16 n a^{2} c^{2} e+18 n a^{2} c d^{2}-(5 n-5) a b^{3} f \\
& +(18 n-16) a b^{2} c e+(3 n-9) a b^{2} d^{2}-(24 n-36) a b c^{2} d+(8 n-16) a c^{4} \\
& -(3 n-3) b^{4} e+(5 n-7) b^{3} c d-(2 n-4) b^{2} c^{3}, \\
C= & 8 n a^{3} c e-9 n a^{3} d^{2}-(4 n-4) a^{2} b^{2} e+(10 n-12) a^{2} b c d \\
& -(4 n-8) a^{2} c^{3}-(2 n-2) a b^{3} d+(n-2) a b^{2} e^{2}, \\
D= & -10 n a^{3} c f+12 n a^{3} d e+(5 n-5) a^{2} b^{2} f-(2 n-8) a^{2} b c e \\
& -(12 n-9) a^{2} b d^{2}+(4 n-12) a^{2} c^{2} d-(n-1) a b^{3} e+(9 n-9) a b^{2} c d \\
& -(4 n-8) a b e^{3}-(2 n-2) b^{4} d+(n-2) b^{3} c^{2},
\end{aligned}
$$




$$
\begin{aligned}
E= & 15 n a^{3} d f-16 n a^{3} e^{2}-(15 n-10) a^{2} b c f+(17 n-12) a^{2} b d e \\
& +(16 n-16) a^{2} c^{2} e-(15 n-18) a^{2} c d^{2}+(5 n-5) a b^{3} f \\
& -(17 n-18) a b^{2} c e+(14 n-24) a b c^{2} d-(n-3) a b^{2} d^{2} \\
& -(4 n-8) a c^{4}+(3 n-3) b^{4} e-(3 n-5) b^{3} c d+(n-2) b^{2} c^{3} \\
F= & -15 a^{2} b d f+16 a^{2} b e^{2}+20 a^{2} c^{2} f-27 a^{2} d^{3}-48 a^{2} c d e \\
& -5 a b^{2} c f+7 a b^{2} d e-4 a b c^{2} e-18 a b c d^{2}+4 a c^{3} d \\
& -3 b^{3} c e+4 b^{3} d^{2}-b^{2} c^{2} d^{2} .
\end{aligned}
$$

Il serait évidemment inutile de vouloir pousser plus loin ces calculs.

[MS. addition in my copy of Liouville.

Quoique ces expressions soient ce qu'il y a de plus simple pour le calcul numérique des fonctions de M. Sturm, cependant sous le point de vue analytique il convient de modifier un peu la forme de ces expressions. En effet en écrivant

$$
V=a x^{n}+\frac{n}{1} b x^{n-2}+\ldots
$$

mettons

$$
\begin{aligned}
& P=a x^{n-1}+\frac{n-1}{1} b x^{n-2}+\ldots,=a x^{n-1}+\theta_{1} b x^{n-2}+\ldots \\
& Q=b x^{n-1}+\frac{n-1}{1} c x^{n-2}+\ldots,=b x^{n-1}+\theta_{1} c x^{n-2}+\ldots
\end{aligned}
$$

l'on aura

$$
\begin{aligned}
& V^{\prime}=n P \\
& U^{\prime}=n Q \\
& V=P x+Q
\end{aligned}
$$

et cela donne après une réduction légère

et ainsi de suite.]

$$
\begin{aligned}
& V_{2}=\left|\begin{array}{cc}
P, & Q \\
a & b
\end{array}\right|, \quad V_{3}=-\left|\begin{array}{cccc}
x P, & P, & x Q, & Q \\
a & . & b & . \\
\theta_{1} b & a & \theta_{1} c & b \\
\theta_{2} c & \theta_{1} b & \theta_{2} d & \theta_{1} c
\end{array}\right|, \\
& V_{4}=\left|\begin{array}{cccccc}
x^{2} P, & x P, & P, & x^{2} Q, & x Q, & Q \\
a & . & . & b & . & \cdot \\
\theta_{1} b & a & . & \theta_{1} c & b & . \\
\theta_{2} c & \theta_{1} b & a & \theta_{2} d & \theta_{1} c & b \\
\theta_{3} d & \theta_{2} c & \theta_{1} b & \theta_{3} e & \theta_{2} d & \theta_{1} c \\
\theta_{4} e & \theta_{3} d & \theta_{2} c & \theta_{4} f & \theta_{3} e & \theta_{2} d
\end{array}\right|
\end{aligned}
$$

